\title{
Computation of flow behind three side-by-side cylinders of unequal/equal spacing
}

\author{
H. K. Virahsawmy* $\quad$ L. Chen ${ }^{\dagger} \quad$ I. R. MacGillivray ${ }^{\dagger}$ \\ J. $\mathrm{Tu}^{*} \quad$ Y. $\mathrm{Zhou}^{\ddagger}$
}

(Received 28 October 2004, revised 10 June 2005)

\begin{abstract}
This paper aims to simulate unstable gap flows of three side-byside cylinders unequally/equally spaced at $T_{1} / d=1.5$ and $T_{2} / d=1.6$ in a uniform cross flow $(\mathrm{Re}=300)$. The simulation is carried out using ANSYs Flotran 7.0. A mesh-independent study is conducted on a single cylinder at $R e=100$. The Strouhal number and wake flow characteristics compare well with experimental results. Simulation of three side-by-side cylinders in uniform cross flow has revealed that the gap flows are unstable and constantly re-orientating, which has
\end{abstract}

*School of Aerospace, Mechanical and Manufacturing Engineering, RMIT University, Australia.

${ }^{\dagger}$ Maritime Platforms Division, DSTO Melbourne, Australia. mailto:Li.Chen@dsto defence.gov . au

${ }_{\ddagger}^{\ddagger}$ Department of Mechanical Engineering, The Hong Kong Polytechnic University, HONG Kong.

See http://anziamj.austms.org.au/V46/CTAC2004/Vira for this article, (c) Austral. Mathematical Soc. 2005. Published July 25, 2005. ISSN 1446-8735 
the effect of restructuring the wake flows behind the cylinders. The flow field for unequal spacing of the cylinders is similar to the equal spacing case for some period of time with a symmetrical near wake. However, at other times, the wake flow is asymmetrical. This agrees well with the visualisation of Wang et al. (2002), in which the gap flows and the wake flow are constantly in transition.

\section{Contents}

1 Introduction

C673

2 Numerical method

C676

3 Results and discussion

C677

3.1 Unequal spacing. . . . . . . . . . . . . . . . . . C677

3.2 Equal spacing . . . . . . . . . . . . . . . . . . . C684

4 Conclusion

C687

References

C688

\section{Introduction}

Flow over bluff bodies is widely encountered in many practical applications. A moving maritime platform, a heat exchanger with cross-flow fluid passages to periodically disrupt the flow, airflow around buildings, and combustors with flame holders are just some of these fundamental and challenging fluid mechanics problems. With heat exchanger design, Liou et al. [6] succinctly demonstrated through their experiments that an oscillating flow resulting from spanwise turbulent vortices behind a circular cylinder near a heated 
wall can significantly enhance the heat transfer along the wall due to periodically sweeping movements of the thermal boundary layer. In sustaining and maintaining the safe operations of a maritime platform, and with heat exchangers in industrial boilers and nuclear reactors, a thorough understanding of the vortex structures could also assist in alleviating problems associated with flow-induced vibration and noise that may compromise the structural integrity (Goyder [3]).

Flow over a cylinder has been studied extensively. It exhibits vastly different behaviour as the Reynolds number, based on the free-stream velocity, cylinder diameter, and kinematic viscosity, increases from zero to large values. In the current investigation, we are interested in the problem of flow interference when three cylinders are placed side-by-side in a steady current. Williamson [8] observed that when more than one body is placed in a fluid flow, the resulting forces and vortex shedding pattern may be completely different from those found at the same Reynolds number. A variety of flow patterns characterized by the behaviour of the wake region may be discerned as the spacing between three circular cylinders is changed.

Ishigai et al. [4] ascribed the gap flow deflection for two cylinders to the Coanda effect. Contrarily, Bearman and Wadcock [1] measured different base pressures behind two side-by-side flat plates and they argued that the gap flow deflection was not caused by the boundary layer separation; instead, it was due to the near-wake phenomenon. Their argument was further supported by the Williamson [8] flow visualisation, which showed a deflected gap flow behind two side-by-side flat plates. It was found from Kim and Durbin [5] that the bi-stability is nominally independent of the Reynolds number. The physical mechanisms behind the formation and stability of a narrow and a wide wake and the explanation of the associated 'flipping' phenomenon has been studied by Chen et al. [2] and Zhou et al. [9].

When three cylinders are arranged as shown in Figure 1, the flow behaviour is characterized by the two ratios $T_{j} / d$, where $T_{j}$ are the centre-tocentre cylinder spacings and $d$ is the cylinder diameter. Flow visualisations 


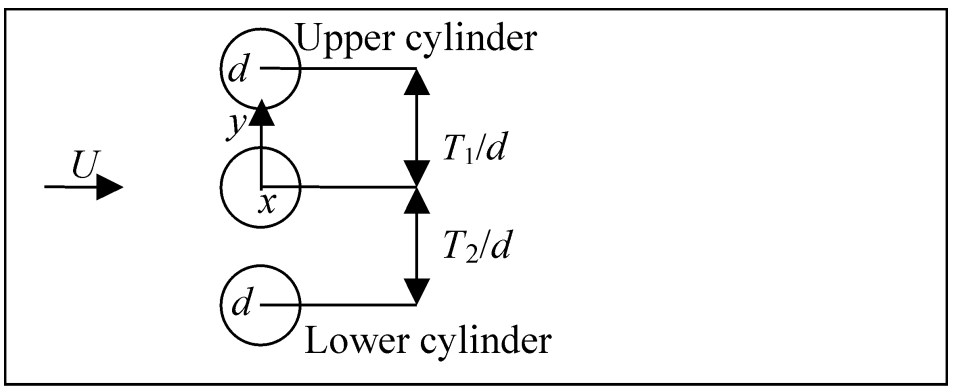

Figure 1: Schematic description of three side-by-side cylinders.

by Williamson [8] and Wang et al. [7] found that with three side-by-side cylinders subject to a uniform cross flow, the wake flow strongly depends upon Reynolds number and the gaps between the cylinders. When the gaps are equal, the flow is symmetric about the centre line, with a wide wake behind the central cylinder and two narrow wakes behind the outer cylinders. However, when the two gaps are different, deflection of the gap flow occurs, forming a wide wake behind the lower/upper cylinder and two narrow wakes behind the upper/lower and central cylinders respectively, depending on the gap spacing. They observed that the deflected gap flows were stable, but non-linear interaction between the two wakes may generate pressure fluctuations that cause re-orientation of both gap flows. This phenomenon is similar to the well-known bi-stable nature of the gap flow deflection that occurs with a two side-by-side cylinder arrangement (Williamson [8]).

The present work deals with a two-dimensional numerical investigation of flows past a three side-by-side circular cylinder configuration. The motivation of this work is driven by the recent experimental observations performed by Wang et al. [7] in the water tunnel facility. They had visualised complex formation of the wake dynamics for a three side-by-side cylinder arrangement with equal and unequal spacing, which allow for direct comparison with the current flow Reynolds number predictions. Our main objectives are: 
1. to validate the phenomenological predictions in the visualisation by Wang et al. [7];

2. to gain a more fundamental understanding of the physical processes within the wake formation region at these two spacings for the flow Reynolds number of 300 .

\section{Numerical method}

The transient simulations were carried out in two dimensions using the finite element technique of the commercial package ANSYS. Prior to the analysis of the three side-by side cylinders, the mesh independent study was carried out on a single cylinder at $\mathrm{Re}=100$ based on the cylinder's diameter, producing results that correlate with the theoretical prediction of vortex shedding around a single cylinder. The validation parameter for the study was the Strouhal number, which is defined as the relationship between the dominant frequency of vortex shedding $f_{s}$, the free stream velocity $U$ and the cylinder diameter $d$ :

$$
\mathrm{St}=\frac{f_{s} d}{U} .
$$

In this study, $d$ is set equal 1. An artificial fluid of density of $1 \mathrm{~kg} / \mathrm{m}^{3}$ and viscosity of $0.01 \mathrm{~kg} / \mathrm{ms}$ was used.

The mesh refinement for subsequent models was applied to the overall domain. In particular, the number of divisions for the $1 \mathrm{~m}$ boundary around the cylinder was refined along the radial axis and boundary line. Three meshes were used in the refinement, and Table 1 gives the corresponding Strouhal numbers. See that as the number of elements increases, the predicted Strouhal number converged to the theoretical value. It is reasonable to assume that a sufficiently accurate solution is obtained by Mesh 3. Therefore, Mesh 3 was used to generate results presented in the following text. 
TABLE 1: Summaries of mesh refinement study

\begin{tabular}{|c|ccccc|}
\hline $\begin{array}{c}\text { Model } \\
\text { number }\end{array}$ & $\begin{array}{c}\text { Number of } \\
\text { divisions } \\
\text { along } \\
\text { Radial axis }\end{array}$ & $\begin{array}{c}\text { Number of } \\
\text { divisions } \\
\text { along } \\
\text { Boundary } \\
\text { line }\end{array}$ & $\begin{array}{c}\text { Total } \\
\text { number of } \\
\text { elements }\end{array}$ & $\begin{array}{c}\text { Strouhal } \\
\text { number }\end{array}$ & $\begin{array}{c}\text { Theoretical } \\
\text { St }\end{array}$ \\
\hline 1 & 12 & 22 & 12056 & 0.1666 & 0.164 \\
2 & 18 & 33 & 26796 & 0.1656 & \\
3 & 24 & 44 & 48224 & 0.1645 & \\
\hline
\end{tabular}

In order to compare with experiment, velocity histories were recorded at 13 different $y / d$ locations ranging between $y / d=3$ to $y / d=-3$ at $x / d=2.5$.

\section{Results and discussion}

\section{$3.1 \quad$ Unequal spacing}

The comparison between the predicted asymmetrical near wake with the experimental observation by Wang et al. [7] is depicted in Figure 2, which is in very good agreement. The predicted evolution of the wake flow behind three unequal spacing cylinders is depicted in Figure 3. However, the arrangement in Figure 3 is unstable and constantly re-orientating, which leads to restructuring of the wake flow behind the cylinders. This constant re-orientation of the gap flows differs from the experimental visualisation (see Figure 2b), in which only changes from one side to another occasionally as a result of non-linear interaction between the wakes.

The periodic nature of the gap flows is shown in Figure 4 by the variation in the angle of deflection, $\theta$, for the upper and lower gap. Both upper and lower gap flows are generally periodic in nature. The lower gap flow has a 


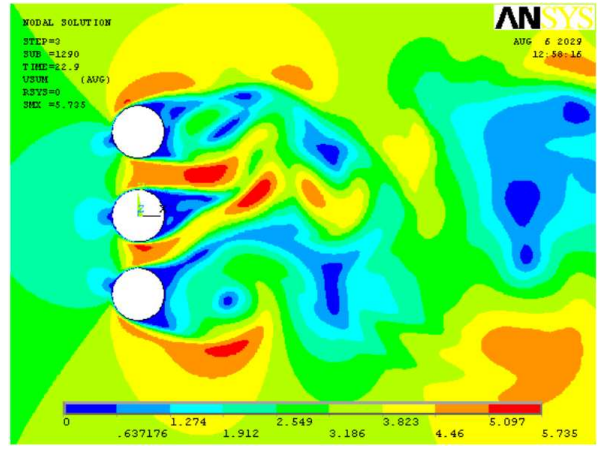

(a) Predicted near wake flow

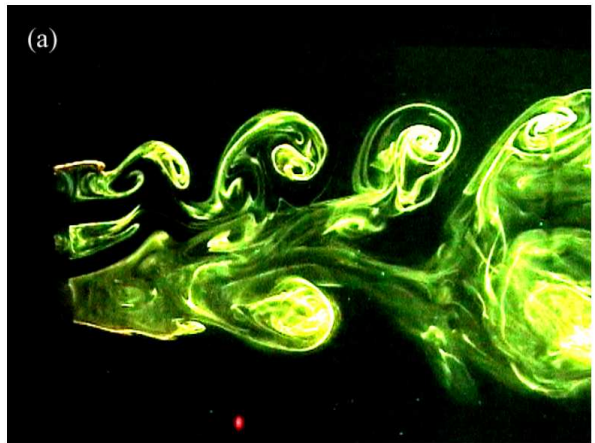

(b) Experimental visualisation

FiguRE 2: Comparison between the predicted near wake with the experimental visualisation by Wang et al. [7] for $\mathrm{Re}=300, T_{1} / d=1.6$ and $T_{2} / d=1.5$.

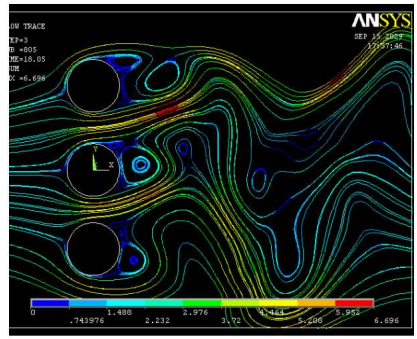

(a) $t=18.1 \mathrm{~s}$

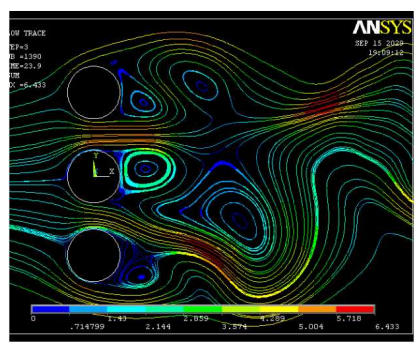

(d) $t=23.9 \mathrm{~s}$

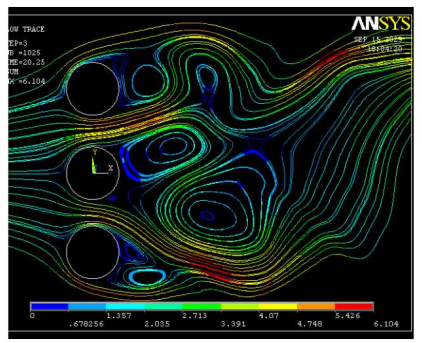

(b) $t=20.3 \mathrm{~s}$

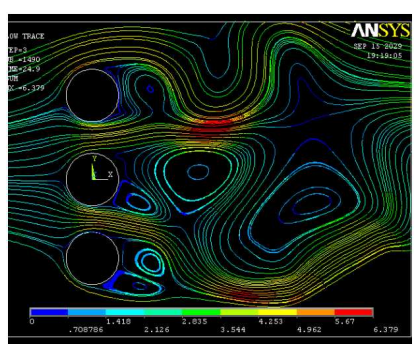

(e) $t=24.9 \mathrm{~s}$

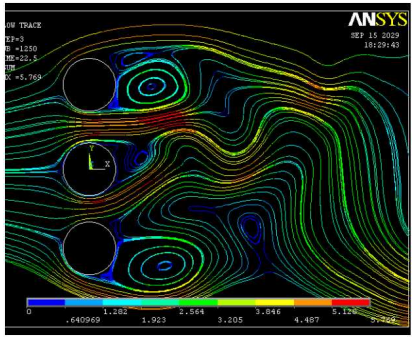

(c) $t=22.5 \mathrm{~s}$

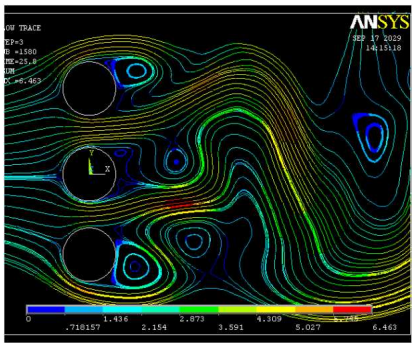

(f) $t=25.8 \mathrm{~s}$

Figure 3: The evolution of the near wake flow over an 8 second period. 


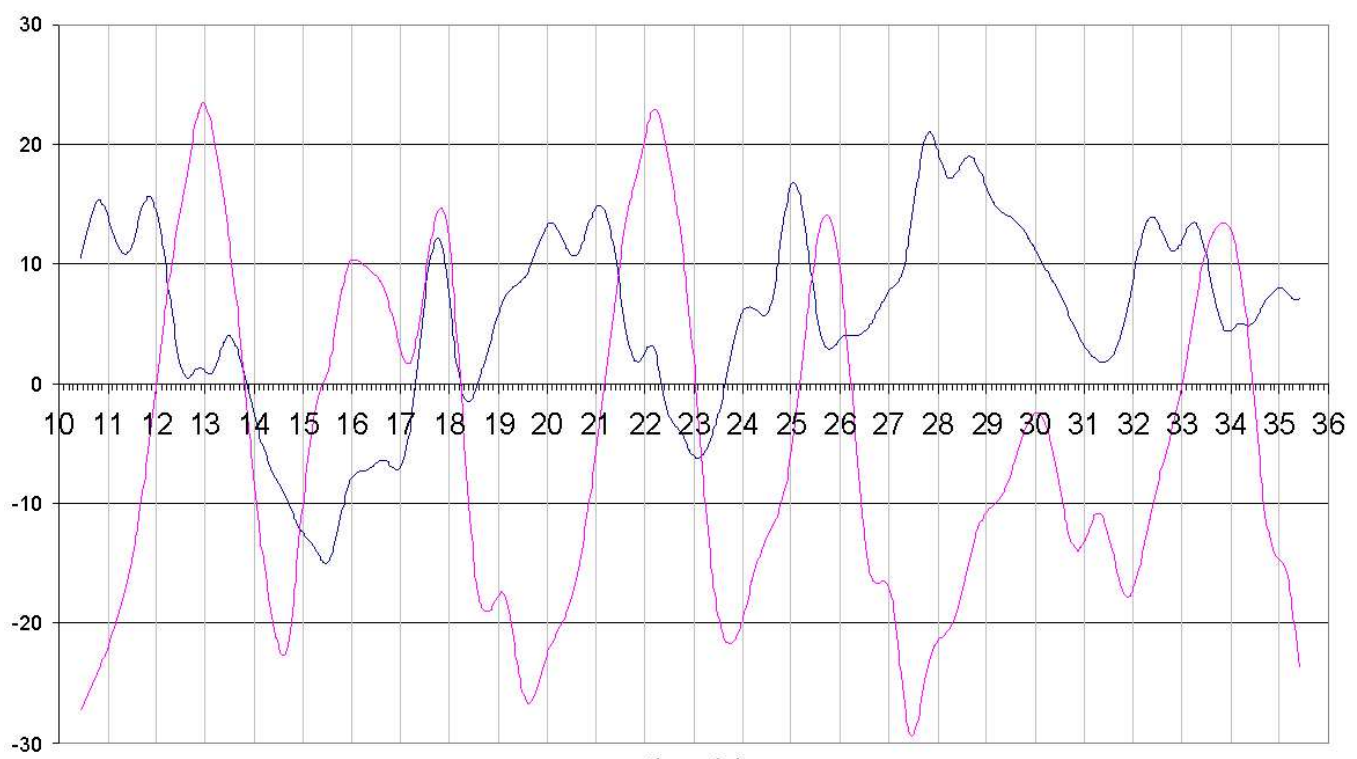

time (s)

\section{- Upper gap flow — Lower gap flow}

Figure 4: Time history of gap flow deflection $\theta$ (in degrees) for unequal cylinder spacing.

larger change of deflection angle, $\Delta \theta$, over time, than the upper gap flow. It also varies between positive and negative inclinations whereas the upper gap flow is positively inclined most of the time.

When the lower gap flow becomes positively inclined, the upper gap flow tends to become less positively inclined. This is observed at the times $t=12$ $14, t=21-23, t=25-26$ and $t=33-34$ seconds. The lower gap flow reaches a maximum positive deflection of 15-20 degrees at which point the upper gap flow is only slightly deflected. The flow field at that instant is similar to the case of unequal spacing observed by Wang et al. [7]. The lower gap flow changes back to a negative inclination while the upper gap flow takes longer to re-orient to a more positive inclination. Hence, the lower gap flow 

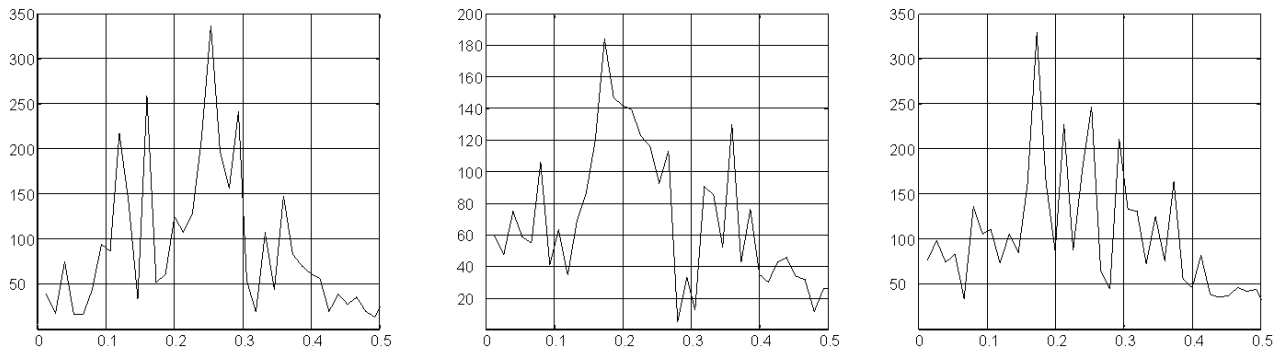

FiguRE 5: Spectrum of lift force for the upper, lower and middle cylinders, respectively. The $x$ axis is the non-dimensional frequency $f^{\star}$.

tends to remain positively inclined for short period of times only. The gap flow deflections as observed by Wang et al. [7] do not occur regularly and are not sustained when they do occur. Rather the gap flows tend to be deflected away from each other most of the time. The lower gap flow fluctuates two times faster than the upper gap flow.

The vortex shedding frequencies from the cylinders were obtained from the lift force variation for the cylinders and are depicted in Figure 5. The spectrum of the lift force histories reveals there are a few modes existing in the evolution of wake flows. It shows one dominant peak at $f^{\star}=f d / U=0.2533$ for the upper cylinder, which is in close agreement with experimental data (Wang et al. [7]). No second harmonic of this frequency was found. The dominant peak for the middle and lower cylinders is at $f^{\star}=0.173$ and its second harmonic was also identifiable but not significant.

Vortices are shed at the same dominant frequency from the middle and lower cylinders at $f^{\star}=0.1734$. Other frequencies including $0.16,0.12,0.36$ for the upper and middle are observed to be harmonics of the upper gap flow normalised frequency, $f^{\star}=0.04$. The gap flow therefore has an influence on the vortex shedding process.

As mentioned by Wang et al. [7], the pressure and velocity fluctuations in 
the near wake as a result of vortex shedding and wake interaction are responsible for the gap flow deflection. The mechanism behind these phenomena needs to be understood. Figure 6 depicts the instantaneous pressure distribution around three cylinders, in which lines outside the cylinder represent negative pressure values and lines inside positive values. A longer outside line represents a more negative pressure value. The pressure fluctuation around the three cylinders is compared in Figure 6 at two times during which both gap flows changes deflection. At $t=19.4 \mathrm{~s}$, the flow is almost symmetric; the lower gap flow has a negative inclination of -20 degrees deflection and the upper gap flow an +8 degrees inclination. At $t=22.4$, the flow is asymmetric; the upper gap flow has a -1.6 degrees deflection and the lower gap flow has a +20 degrees deflection.

As seen in Figure 6a, when the gap flows are deflected away from each other, a shift of the front stagnation points for the upper and lower cylinder was observed and the upper cylinder and lower cylinder are subjected to a repelling force. As expected, the base pressure behind the middle cylinder is higher because of a low momentum, whereas the base pressure behind the upper and lower cylinder will be lower because of the higher momentum as the gaps flows are deflected away from it. As a consequence, symmetrical wake streets are obtained. However, the forces acting on three cylinders are different when the asymmetrical wake occurs (Figure 6b). When the flow is asymmetric, the base pressure behind the middle cylinder becomes significantly lower. The base pressure behind the upper cylinder almost matches the middle cylinder, explaining the small defection of the upper gap flow. The base pressure of the lower cylinder is higher than the middle cylinder as the lower gap flow is deflected away from it. The pressure changes are therefore observed to be significant for each cylinder as the gap flows re-orient.

The spectra measured at different $y / d$ locations show that for $1.5 \leq$ $y / d \leq 3$, the dominant frequency of the flow is $f^{\star}=0.253$, as shown in Figure 7, which is in close agreement with experimental data (Wang et al. [7]). This peak indicates the dominant vortex frequency from the upper cylinder. 

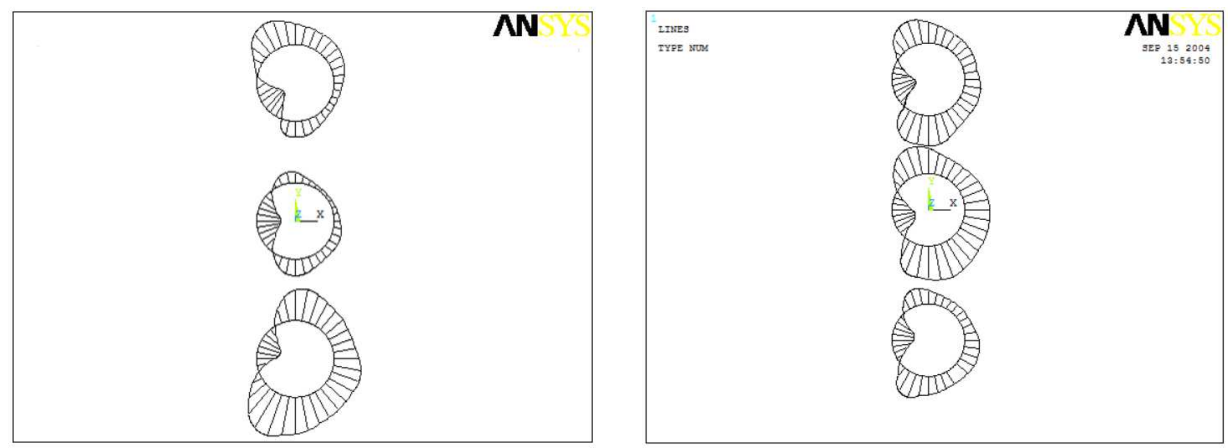

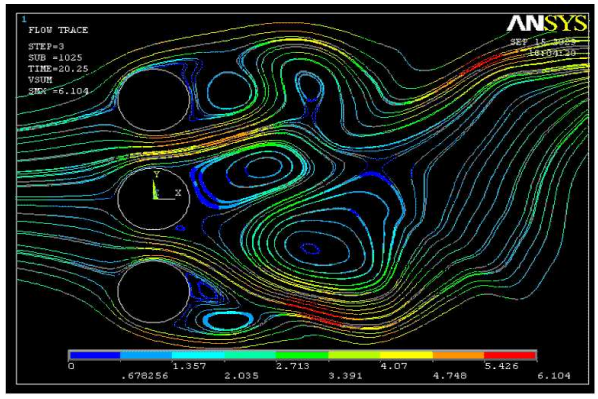

(a) Symmetrical, $t=19.4 \mathrm{~s}$

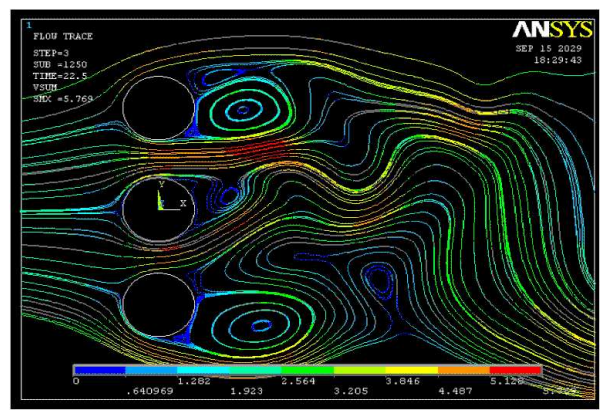

(b) Asymmetrical, $t=22.4 \mathrm{~s}$

FiguRE 6: Instantaneous pressure distributions and gap flows around the three unequally spaced cylinders.

The next dominant peak is at $f^{\star}=0.16$ and its second harmonic was also identifiable but not significant. These frequencies indicate vortex frequencies from the upper cylinder.

For $-3 \leq y / d \leq 1$, the dominant frequency at $f^{\star}=0.08$, as in Figures $7 \mathrm{~b}$ to $7 \mathrm{c}$, probably indicates a single vortex shedding frequency. This frequency is in close agreement with that observed by Wang et al. [7] at $f^{\star}=0.076$, which was dominant across the wake further downstream. The rolling up of shear layers into vertical structures was claimed to be responsible for the peak instead of originating from the vortex shedding process. This is confirmed in our case as the peak $f^{\star}=0.08$ is not detected as a vortex shedding frequency 


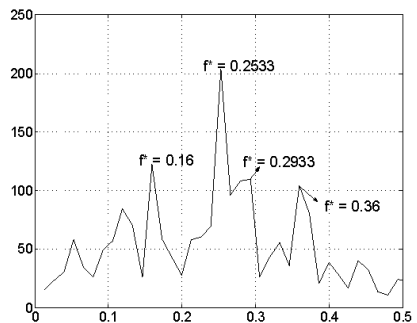

(a)

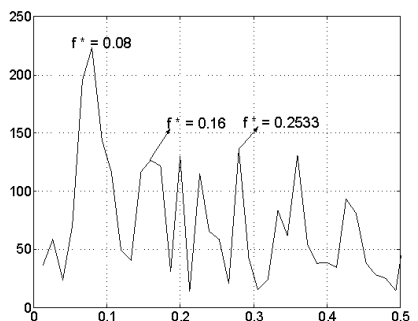

(b)

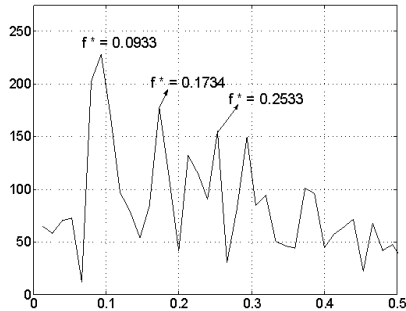

(c)

Figure 7: Power Spectra $E$ at $x / d=2.5$, (a) $y / d=2.5$, (b) $y / d=0.5$. The $x$ axis is the non-dimensional frequency $f^{\star}$.

from the cylinders using the lift force.

However, the less dominant peaks in Figures $7 \mathrm{~b}$ to $7 \mathrm{c}$ indicate vortexshedding frequencies. For $-0.5 \leq y / d \leq 1, f^{\star}=0.253$ indicates the upper cylinder vortex frequency. The middle cylinder dominant vortex frequency of $f^{\star}=0.173$ is only identifiable at $y / d=0.2$. The lower cylinder vortex frequencies $f^{\star}=0.173$ and $f^{\star}=0.253$ are identified at $-3 \leq y / d \leq-1.5$ (Figure $7 \mathrm{c}$ ). Vortex shedding from the upper is therefore clearly dominant in the wake for $y / d \geq 1.5$. However, the lower side of the wake is clearly dominated by some sort of vortical structure at $f^{\star}=0.08$.

Hence observe that although the flow pattern is constantly changing, it bears similarity with the Wang et al. [7] stable asymmetric flow in terms of dominant frequencies across the near wake. In particular, the identification of a similar single vertical structure as Wang et al. [7] observed is interesting despite the fact that gap flow re-orientation is observed in our case, rather than shear layer rolling. 


\subsection{Equal spacing}

The flow visualisation by Wang et al. [7] is shown in Figure 8, which demonstrates a symmetrical wake flow about the centre line of the middle cylinder for the equal spacing configuration. However, the simulation, shown in Figure 9, predicts that the gap flows are unstable and constantly re-orientating, as was the case with the unequal spacing configuration.

Both upper and lower gap flows are again generally periodic in nature. In contrast to the unequal spacing configuration, the lower gap and upper gap flows in this case have almost the same change in angle deflection, $\Delta \theta$, over time. Both gap flows also vary between positive and negative inclinations.

The symmetric flow observed by Wang et al. [7] occurs only initially in the predicted results at $t=10-12 \mathrm{~s}$. As with the unequal spacing configuration, when the lower gap flow becomes positively inclined, the upper gap flow immediately responds to a less positive inclination. However, when the lower gap flow becomes negatively inclined again, the upper gap flow does not deflect back upward as in the unequal spacing case. Hence the symmetric flow is not observed again in Figure 9. Instead the gap flows are either deflected towards each other or in the same direction. It seems that the lower gap flow fluctuates four times faster than the upper gap flow. The upper gap flow reacts more slowly to the lower gap flow re-orientation.

As with the unequal spacing configuration, the lift force on the upper cylinder is mostly positive, that of the middle cylinder varies between relatively smaller positive and negative values, and that of the lower cylinder is mostly negative. Hence the upper and lower cylinders are repelled from each other in both spacing configurations. The pressure fluctuations from the vortex shedding must be in this case large enough to cause the gap deflection to change over. The pressure distributions around the cylinders at two different times are shown in Figure 10. At $t=11.2$, the flow is symmetric, and as expected, the upper and lower cylinders have lower base pressure than the middle cylinder and pressure distributions around three cylinders 


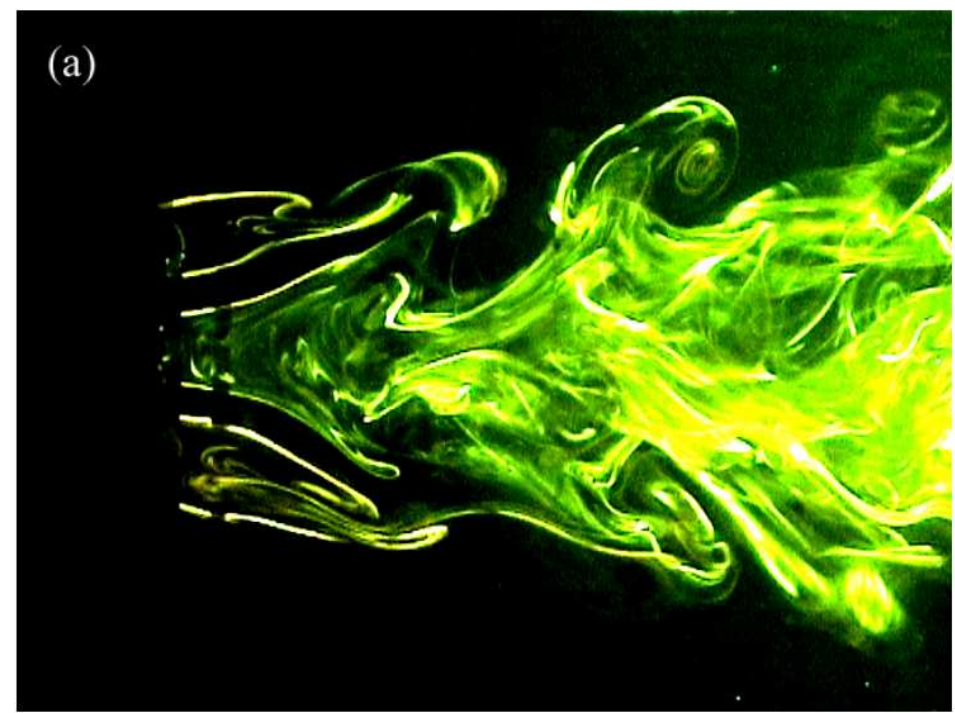

Figure 8: Experimental visualisation at $\operatorname{Re}=300, T_{1} / d=T_{2} / d=1.5$ by Wang et al. [7].

are symmetric about the centerline. At $t=13.9$, the base pressures of the upper and middle cylinders are almost equal and the base pressure of the lower cylinder is higher than that of the middle cylinder. Hence as expected, the asymmetric vortex streets were obtained, in which the upper gap flow is almost not deflected and the lower gap flow is positively inclined.

The vortex frequencies derived from the lift force histories reveal there are a few modes of vortex shedding existing as shown in Figure 11. The dominant frequencies of vortex shedding are observed to be harmonics of the upper gap flow frequency, $f^{\star}=0.0329$. The gap flow re-orientation is therefore more strongly associated with the vortex shedding from the cylinders, in with equal spacing than with unequal spacing.

Comparison of the spectra reveals that vortices are shed from the middle and lower cylinders with better defined frequencies than the upper cylinder, 


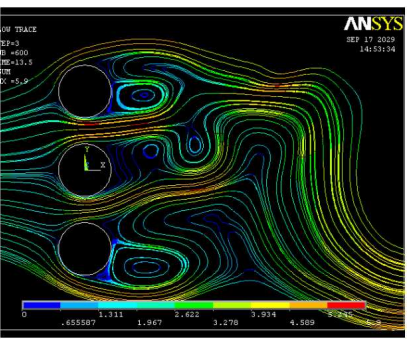

(a) $t=13.5 \mathrm{~s}$

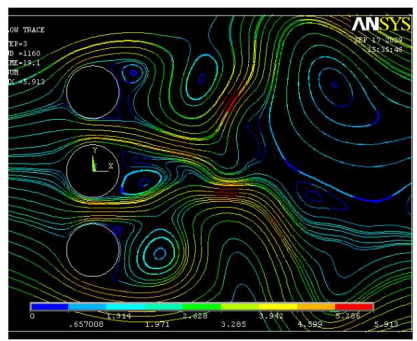

(d) $t=19.1 \mathrm{~s}$

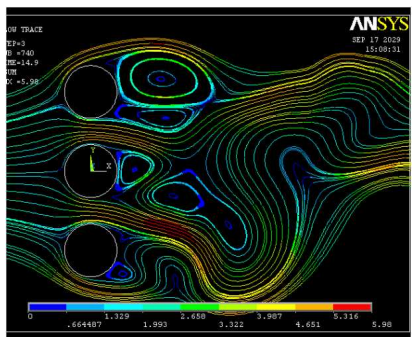

(b) $t=14.9 \mathrm{~s}$

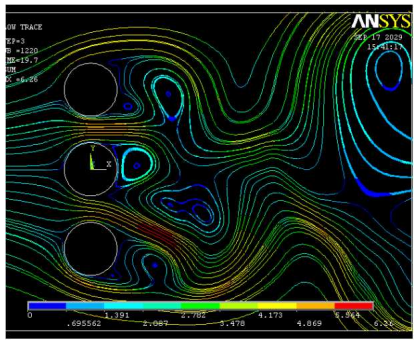

(e) $t=19.7 \mathrm{~s}$

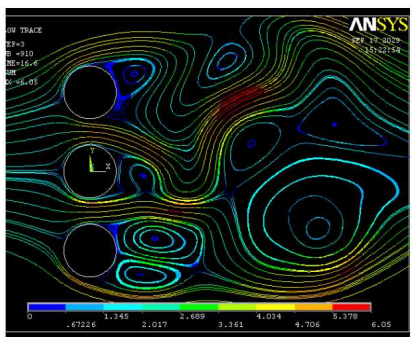

(c) $t=16.9 \mathrm{~s}$

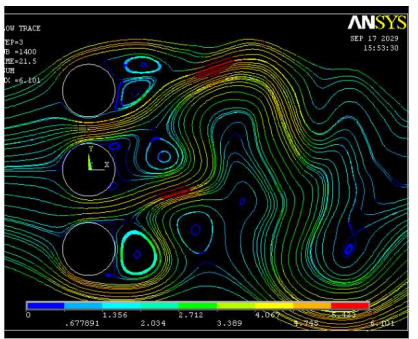

(f) $t=21.5 \mathrm{~s}$

FiguRE 9: Evolution of wake flow for equally spaced cylinders and with higher strength.

The spectra measured at $x / d=2.5$ display a major peak at $f^{\star}=0.0823$ close to the flow centre line $(-1 \leq y / d \leq 1)$ which is close in agreement with the frequency of $f^{\star}=0.069$ recorded by Wang et al. [7] at the same locations. This frequency is consistent with the upper gap flow re-orientation frequency. The re-orientation of the gap flows is therefore strongly influential in the middle section as with the unequal spacing configuration. For $y / d>-1.5$, the dominant frequency is $f^{\star}=0.132$. Its second harmonic, $f^{\star}=0.263$, is less prominent but still identifiable. These frequencies are consistent with both the lower gap flow re-orientation frequency and the lower-cylinder vortex frequency. Hence it is again observed that the gap flows and vortex shedding are associated in some way. For $1.5 \leq y / d \leq 2$, the dominant peak at $f^{\star}=0.132$ is again observed. It indicates a vortex frequency from the upper 


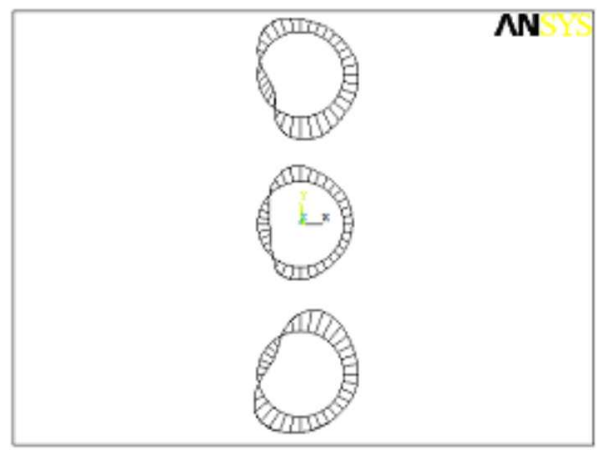

(a) Symmetrical, $t=11.2 \mathrm{~s}$

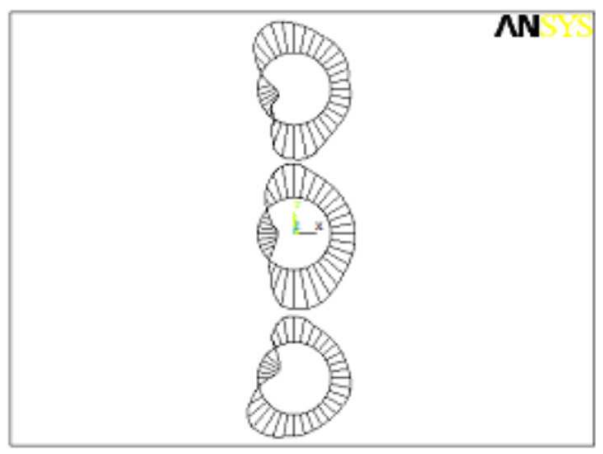

(b) Asymmetrical, $t=13.9 \mathrm{~s}$

FiguRE 10: Instantaneous pressure distribution around the three cylinders.

cylinder. Its second harmonic is also identified at $f^{\star}=0.263$ at $y / d=1.5$. Unlike the unequal spacing configuration, the upper gap flow frequency is still identifiable at $y / d$ greater than 1.5 .

Hence observe that the middle section is again dominated by gap flow re-orientations. But unlike the unequal spacing case, the gap flows are more in control of the upper and lower vortex shedding process, and therefore the upper and lower wake tend to possess the same frequencies.

\section{Conclusion}

The computational method has shown that the stable flow observed by Wang et al. [7] for the equal and unequal spacing configurations are observed in the simulation. However, the wake flow in either case is constantly restructuring as the gap flows are not stable.

Nevertheless, observe that the wake flow bears similarities with the experimental observations in both cases. Similar frequencies are identified in the near wake. In particular, the detection of vertical structures that do not 


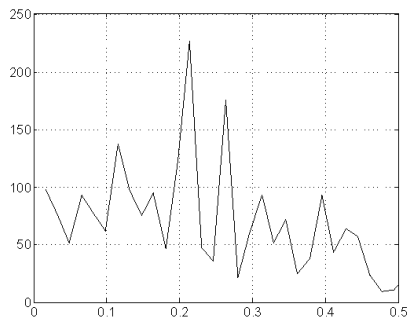

(a)

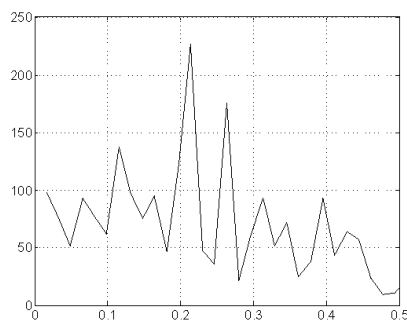

(b)

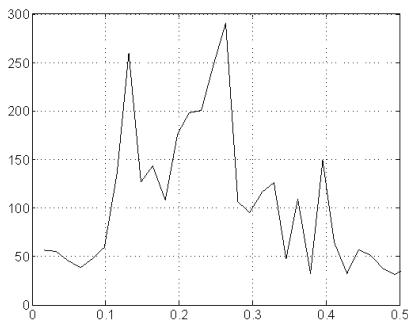

(c)

Figure 11: Power Spectra E: (a) upper cylinder, (b) middle cylinder, (c) lower cylinder. The $x$ axis is the non-dimensional frequency $f^{\star}$.

originate from the shedding process is important. Wang et al. [7] suggested rolling of shear layers as responsible for the vertical structures; however, this is not observed in the flow simulation. Rather we propose, based on observations, that the process of gap flow re-orientation generates vertical structures that may explain the same frequencies detected as that of the rolling-up.

Further computational work will be carried out to reinforce the above conclusions. In particular, we expect that running the simulation for longer will provide more accurate flow frequencies and better agreement between gap flow re-orientation phenomenon and the experimentally stable flow. Mesh refinement will also be carried out to reduce numerical error. Once the flow model is validated, the study will also be extended to evaluate flow-structure interaction.

\section{References}

[1] P. W. Bearman, A. J. Wadcock. The interaction between a pair of circular cylinders normal to a stream, Journal of Fluid Mechanics, 61, 1973, 499-511. C674 
[2] L. Chen, J. Y. Tu and G. H. Yeoh. Numerical simulation of turbulent wake flows behind two side-by-side cylinders, Journal of Fluids and Structures, 18, 2003, 387-403. C674

[3] H. G. D. Goyder. Flow-induced vibration and noise in heat exchanger tube bundles, 12th International Heat Transfer Conference, Grenoble, France, 2002. C674

[4] S. Ishigai, E. Nishikawa, K. Nishmura and K. Cho. Experiment study on structure of gas flow in tube banks with tube axes normal to flow (Part I, Karman vortex flow around two tubes at various spacings), Bulletin of the JSME, 15, 1972, 949-956. C674

[5] H. J. Kim and P. A. Durbin. Investigation of the flow between a pair of cylinders in the flopping regime, Journal of Fluid Mechanics, 196, 1988, 431-448. C674

[6] T. M. Liou, W. B. Wang and Y. J. Chang. Holographic interferometry study of spatially periodic heat transfer with ribs detached from one wall, Journal of Heat Transfer, 117, 1995, 32-39. C673

[7] W. Wang, H. J. Zhang, Y. Zhou and J. Y. Tu. Flow visualisation behind three cylinders of equal and unequal spacing, International Journal of Flow Visualisation and Image Processing, 9, 2002, 139-151. C675, C676, C677, C678, C679, C680, C681, C682, C683, C684, C685, C686, C687, C688

[8] C. H. K. Williamson. Evolution of a single wake behind a pair of bluff bodies, Journal of Fluid Mechanics, 159, 1983, 1-18. C674, C675

[9] Y. Zhou, Z. J. Wang, R. M. C. So, S. J. Xu and W. Jin. Free vibrations of two side-by-side cylinders in a cross flow, Journal of Fluid Mechanics, 443, 2001, 197-229. C674 